\title{
Fuzzy Clustering Based Bayesian Framework to Predict Gynaecological Problems among girls
}

\author{
K.A.Anjana \\ Department of Computer Science, \\ Bhaktavatsalam Memorial College for Women, India \\ E-mailanjana.gujula@gmail.com
}

\begin{abstract}
Young girls experiencing gynaecological problems disturbs their physical and mental wellbeing. Heavy periods, Amenorrhea, Dysmenorrhea, Menorrhagia, Oligomenorrhea, Endometriosis, PCOD are some of the common problems by young girls. Untreated they cause anaemia and other issues. When they reach child bearing age the have trouble conceiving which affects their families. Predicting the problems we could avoid them and help the individual lead a happy life. Some cases these gynaecological problems manifest into cancer. We predict gynaecological problems using our fuzzy clustering based Bayesian framework.
\end{abstract}

Keywords : Bayesian Framework, Gynaecological, fuzzy clustering

I

\section{. INTRODUCTION}

Gynaecological problems are most prevelant in young girls. Dysmenorrhea, PCOD, Endometriosis, ovarian cysts are some the most common prevelant gynaecological problems. These problem affects the performance of women and also leads to other health problems. Due to these problems there is absenteeism in schools, colleges, work place and they are not fully productive due to these reasons.

Its best that we can find out girls who are likely to face the problem at the earliest so their quality of life improves and also they could lead a happy life in future.

\section{RELATED WORK}

Now-a-days breast cancer is one the most common cancer in the world. Its really important that we diagnose at a higher rate of accuracy. Patients cytological images obtained during fine needle aspiration biopsy are classified[1].

Prediction of mental health problems among children using fuzzy clustering Bayesian framework help in identifying it, even before it occurs. The quality of lives of the children are greatly impacted and they could focus on their education and also lead a happy life.

Bayesian Network

They are very effective in computation and representation of random variables and their combined probabilistic distribution. The nodes represent random variables and the edges represents probabilistic dependencies. 


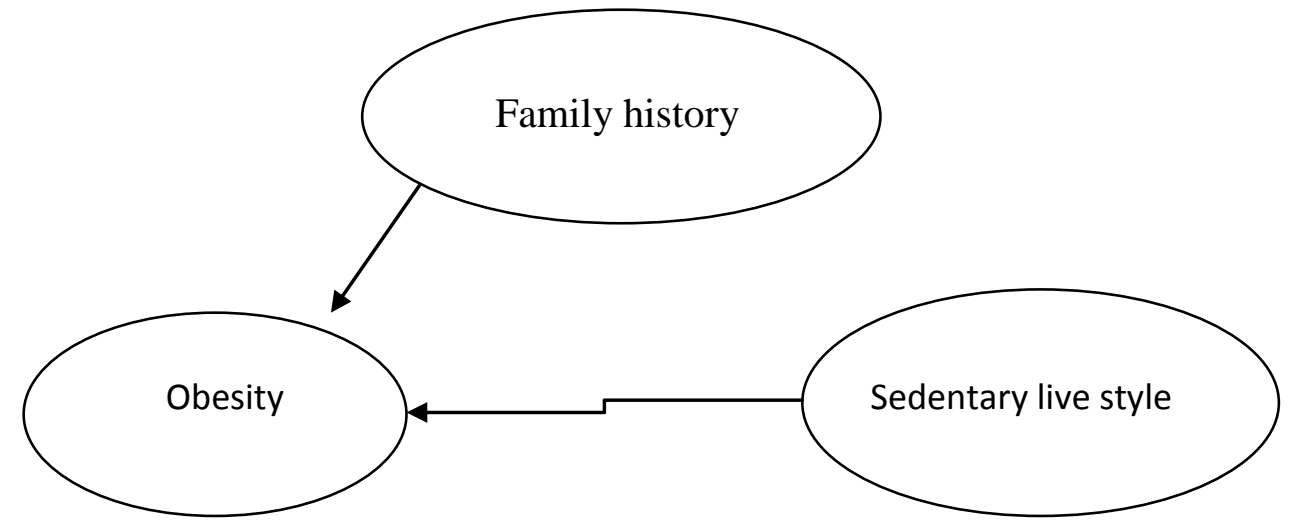

\section{Fuzzy clustering}

Clustering involves classification of data points into different groups or clusters. The data points in the same cluster have same characteristics or they are similar, whereas the data points in different clusters are dissimilar. We have two types of cluster determination methodologies one is nonfuzzy systems and the other one is fuzzy systems. Non-fuzzy systems groups data objects with strict boundaries. The problem is that the data points that near the boundary tend to get a different cluster is the boundary is slightly altered. To take in account the data points of this kind fuzzy systems are used where a data point which is close two clusters get appropriate weighed.

\section{Fuzzy c-means}

Fuzzy c-means is the fuzzy version of k-means algorithm. We initialise the cluster and centroid. FCM repeatedly calculates the centroid of the cluster and the fuzzy pseudo-partition is updated using new values. The same procedure is repeated until the partition does not change.

\section{PROPOSED FRAMEWORK}

The proposed framework consist of the following steps. In the first phase the relevant documents are collected. Then the document is pre-processed i.e filling of missing fields, removal of documents which are incomplete. Attributes are selected and their values are extracted. Our proposed fuzzy clustering based Bayesian Network structure learning algorithm is used to predict gynaecological problems among girls.

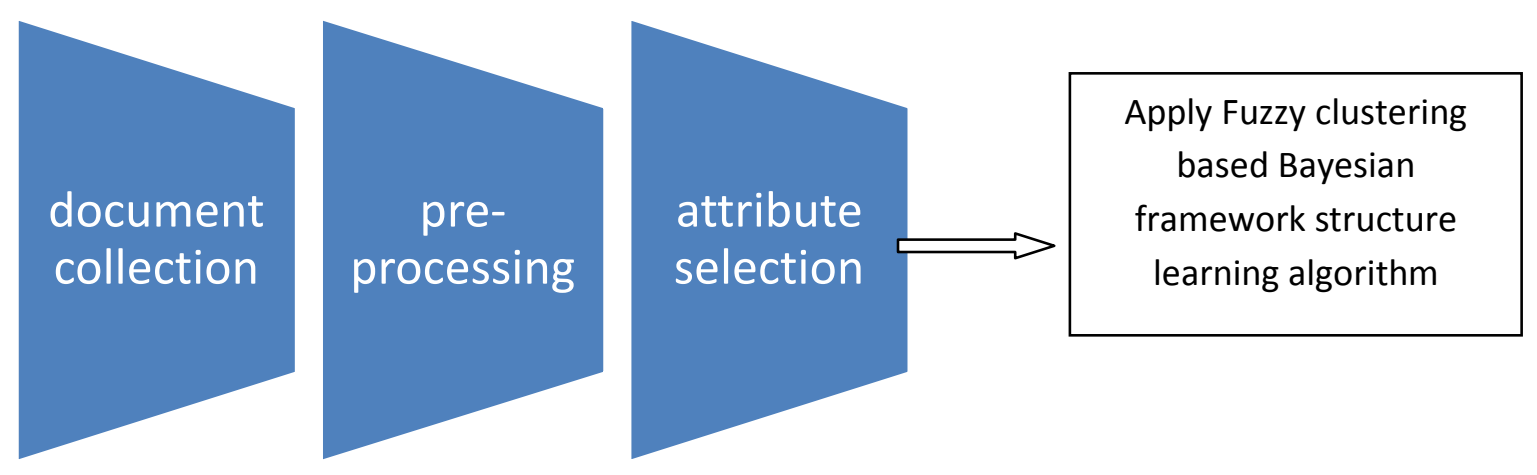

Vol 1 (8), February 2018, www.ijirase.com 
Fuzzy clustering based Bayesian Network Structure Learning Framework(FCBN)

Basic algorithm of fuzzy c-means:

1. Select an initial nodes that are mutual.

2. Based on mutual information, group the nodes into various clusters using Fuzzy Clustering techniques.

3. Using scoring function build Bayesian network

4. Based on the fuzziness of nodes, build a complete Bayesian network by combining several local Bayesian networks.

Grouping of nodes based on mutual information among nodes

Bayesian network is composed of nodes and edges. The edges represent the dependencies between the nodes. The dependencies between the nodes can be identifies using the following equation.

$\mathrm{I}(\mathrm{A}, \mathrm{B})=\sum \mathrm{P}(\mathrm{a}, \mathrm{b}) \log \frac{P(a, b)}{P(a) P(b)}$

Using the mutual information and if they are dependent they are determined how close their relationship is they are grouped together. If the number of variables are large the network grows exponentially so they are grouped together.

Constructing and combing the network
Initially the $\mathrm{n}$ variables are divided into $\mathrm{k}$ clusters and the basic network is formed. After Building the network if the same variable say ' $\mathrm{x}$ ' is found in both BN1 and BN2 networks, fuzzy nodes are identified to combine the network.

\section{IV.CONCLUSION}

FCBN could be used the predict the gynaecological problems of girls in a effective manner. Future complications due to gynaecological problems which would manifest into severe illness could be avoided by predicting and treating it at the first place. We get the information from the various attributes responsible for it and address the issue.

"Prevention is better than cure".

\section{REFERENCES}

1.https://yadda.icm.edu.pl/baztech/element/b wmeta1.../Brudzinski_web_based.pdf

2.ictactjournals.in/paper/IJSC_Vol_7_Iss_4_ Paper_4_1452_1458.pdf by MR Sumathi and B.Poorna.

3.https://www.ncbi.nlm.nih.gov/pmc/articles /PMC5043229/ 